\title{
Parameter optimization for the three-frequency feed for the mirror antenna of the non-linear radar
}

\author{
Vitaly Slyozkin ${ }^{1, *}$ and Mikhail Nevedrov ${ }^{1}$ \\ ${ }^{1}$ Sevastopol State University, Radio Electronics and Telecommunications Chair, 299053, \\ 33 Universitetskaya St., Sevastopol, Russia
}

\begin{abstract}
Analyzed below is a radiating structure represented by a flat self-complementary three-sector structure fed by three segments of the microstrip line and located above the stepped metal screen, and to be used as an exciter for the non-linear radar antenna. When proper matching with the feeding coaxial cable is achieved, results of the numerical simulation proved that the antenna can provide near-circular polarization of radiation at the probing signal frequency, and linear polarization at the second and third harmonics of this signal.
\end{abstract}

\section{Introduction}

Non-linear radars used for locating concealed radio devices illegally picking up information are most commonly fitted with mirror antennas with three-frequency exciters. Probing signal is transmitted by the field with circular polarization at the lowest frequency $f_{1}$ while signals at second and third harmonic frequencies $\left(f_{2}=2 f_{1}\right.$, and $f_{3}=3 f_{1}$, respectively) are received through linear polarizations. It is quite difficult to design such exciters therefore research in this field is reasonable.

This report analyzes feasibility of a three-frequency feed based on a modified flat selfcomplementary structure with a stepped screen.

\section{The object under consideration and the methodology of its investigations}

Flat self-complementary structures are well known and they retain their shape when the metal strips are replaced with vacuum and vice versa [1] therefore they can operate as band antennas with frequency overlap ratio of $1: 3$ and higher. However, firstly their impedance is close to $180 \mathrm{Ohm}$ which makes it difficult to match it with 50-Ohm cable and secondly they have quasi-isotropic patterns.

To improve matching conditions, angles between metal sectors should be reduced [2] while making corner cutouts and connecting edges of the sectors with narrow jumpers [3]

*Corresponding author: slezkinvg@mail.ru 
in order to retain broadbandness. Circular polarization of radiation at one frequency can be achieved in three-sector structures having feeding circuits based on the microstrip lines (Fig. 1).

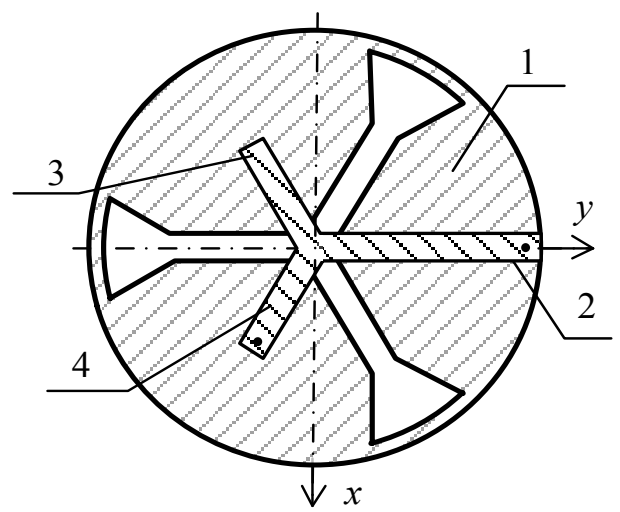

Fig. 1. Radiating structure arrangement.

The flat structure 1 consisting of the three sectors (see Fig. 1) is implemented on the lower (relative to the radiation direction) surface of the dielectric base (not shown in the Figure). The upper surface of the base includes leading-in segment of the microstrip line (MSL) 2, open 3 and short-circuited 4 segments of the MSL.

Since segments 3 and 4 have reactances at frequency $f_{1}$ they can be substituted on the equivalent schematic with a capacitance $\mathrm{C}$ and inductance L, respectively (Fig. 2).

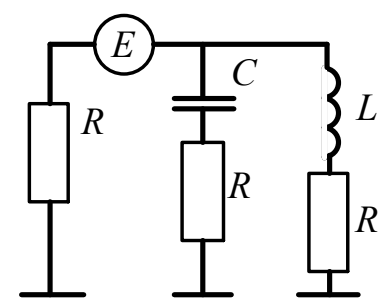

Fig. 2. Equivalent schematic of the feeding circuit.

To achieve circular polarization it is sufficient that segments 3 and 4 created phase shifts of $-60^{\circ}$ and $+60^{\circ}$. By varying the shape of the radiating structure it is easy to achieve purely active impedance $R$ of each sector, therefore capacitance $C$ of the open segment 3 and inductance $L$ of the short-circuited segment 4 shall meet obvious conditions:

$$
\frac{2 \pi f_{1} L}{R}=\frac{1}{2 \pi f_{1} C R}=\tan 60^{\circ}=\sqrt{3}
$$

By using known relations for closed and open segments of the feeder [1], we will get

$$
\frac{\rho_{3} \operatorname{ctg} k l_{3}}{R}=\frac{\rho_{4} \operatorname{tg} k l_{4}}{R}=\sqrt{3} .
$$


where $l_{3}, l_{4}$ - lengths of MSL segments 3 and 4;

$\rho_{3}, \rho_{4}$ - wave impedances of these segments;

$k$ - wave factor.

Since this is preferred (but not mandatory) that wave impedances of all MSL segments are equal, from (2) it follows that length of the segments will be equal to

$$
\frac{l_{3}}{\lambda}=0,083 ; \quad \frac{l_{4}}{\lambda}=0,167
$$

Unidirectional radiation can be achieved by using a flat metal screen of a large enough diameter that is installed at a distance $h_{1}$ being close to one quarter of the wave length in the free space. However at frequencies $f_{2}$ and $f_{3}$ the screen influence can result in mismatching of the feed and the feeding cable. To make is possible to independently adjust impedance at these frequencies, we can try to use a stepped screen with distances $h_{2}, h_{3}$ being close to one quarter of the wave length at their frequencies (Fig. 3).

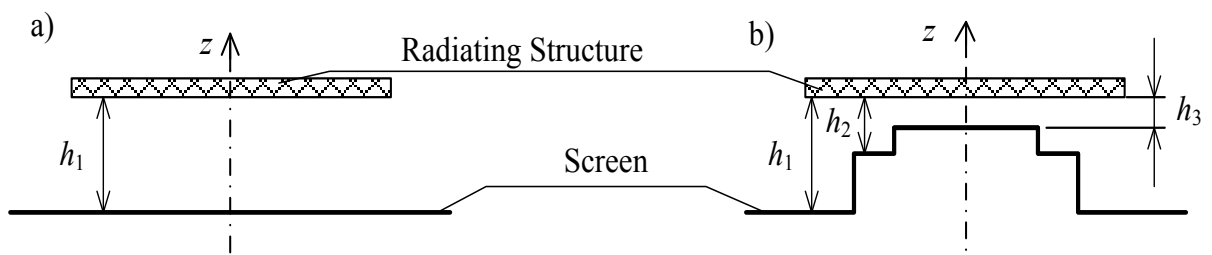

Fig. 3. Feed schematic with a flat (a) and stepped screen (b).

To ensure that above considerations are reasonable, a numerical simulation of the antenna was performed in the CAD system CST Microwave Studio (MWS). Initial values of the design radius of the structure and distance to the screen were close to one quarter of the wave length for the lowest frequency $f_{1}$ equal to $2400 \mathrm{MHz}$. Preliminary values of the MSL segment lengths were selected based on (3). By varying the principal dimensions of the model it was possible to achieve input matching at standing wave ratio of less than 2.0 in relative frequency bands (around each of the design frequencies) not less than 3\%, with ellipticity of radiation at frequency $2400 \mathrm{MHz}$ of less than 0.7 , and aperture angles at $-10 \mathrm{~dB}$ level of the model at frequencies $2400 \mathrm{MHz}, 4800 \mathrm{MHz}$ and $7200 \mathrm{MHz}$ being $66^{\circ}$, $46^{\circ}$ and $52^{\circ}$, respectively. The level of lateral radiation at all frequencies did not exceed $-9 \mathrm{~dB}$.

\section{Conclusion}

Based on the results of the numerical simulation in CST MWS system it was shown that a three-frequency feed for the non-linear radar antenna based on the flat self-complementary structure and stepped metal screen is feasible.

\section{References}

1. A. Fradin Antenna-feeder devices (Svyaz, Moscow, 1977)

2. B. Mishoostin, V. Slyozkin, Conference EuCAP-2012, pp. 3848-3851 (2012)

3. B. Mishoostin, V. Slyozkin, Conference Cri-MiCo-2016, 5, pp. 965-970 (2016) 